\title{
Anti-citrullinated protein antibodies acquire a pro-inflammatory Fc glycosylation phenotype prior to the onset of rheumatoid arthritis
}

\author{
Yoann Rombouts, ${ }^{1}$ Ewoud Ewing, ${ }^{2}$ Lotte A van de Stadt, ${ }^{3}$ Maurice H J Selman, ${ }^{2}$ \\ Leendert A Trouw, ${ }^{1}$ André M Deelder, ${ }^{2}$ Tom W J Huizinga, ${ }^{1}$ Manfred Wuhrer, ${ }^{2}$ \\ Dirkjan van Schaardenburg, ${ }^{3,4}$ René E M Toes, ${ }^{1}$ Hans U Scherer ${ }^{1}$
}

\begin{abstract}
Handling editor Tore K Kvien
- Additional material is published online only. To view please visit the journal online (http://dx.doi.org/10.1136/ annrheumdis-2013-203565).

${ }^{1}$ Department of Rheumatology, Leiden University Medical

Centre, Leiden,

The Netherlands

${ }^{2}$ Center for Proteomics and Metabolomics, Leiden University Medical Centre, Leiden, The Netherlands ${ }^{3} J a n$ van Breemen Research Institute | Reade, Amsterdam, The Netherlands

${ }^{4}$ Department of Rheumatology, VU University Medical Centre, Amsterdam, The Netherlands
\end{abstract}

\section{Correspondence to}

Dr H U Scherer,

Department of Rheumatology, Leiden University Medical Centre, P.O. Box 9600 Leiden 2300 RC, The Netherlands; h.u.scherer@|umc.n|

Received 5 March 2013 Revised 31 July 2013 Accepted 17 September 2013 Published Online First 8 October 2013

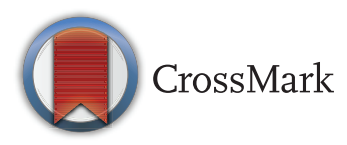

\footnotetext{
To cite: Rombouts $Y$, Ewing $E$, van de Stadt $L A$, et al. Ann Rheum Dis 2015;74:234-241.
}

\section{ABSTRACT}

Objective To determine whether anticitrullinated protein antibodies (ACPA) exhibit specific changes in

Fc glycosylation prior to the onset of arthritis.

Methods Serum samples of patients with ACPA-positive arthralgia ( $n=183$ ) were collected at baseline and at various time points of follow-up. 105 patients developed arthritis after a median of 12 months (IQR 6-24) and were classified as having either rheumatoid arthritis (RA, $n=48$ ) or undifferentiated arthritis (UA, $n=57)$ based on the 1987 American College of Rheumatology (ACR) criteria. ACPA and total serum lgG were isolated by affinity purification and cleaved by trypsin. ACPA-IgG1 Fc-glycopeptides were subsequently analysed by nano-liquid chromatography mass spectrometry and compared to those of total IgG1. Results At baseline, ACPA-IgG1 and total IgG1 from arthralgia patients displayed similar Fc glycosylation patterns. By contrast, at the onset of arthritis, ACPA exhibited a decrease in galactose residues in RA patients, but not in UA patients. This decrease occurred around 3 months prior to diagnosis and was paralleled by an increase in systemic inflammation (erythrocyte sedimentation rate). Galactosylation of total $\lg G 1$ was also decreased in RA, but this did not precede the onset of arthritis. Interestingly, we additionally noted a higher degree of ACPA-IgG1 Fc core fucosylation at baseline as compared with total $\lg G 1$, which further increased prior to diagnosis.

Conclusions ACPA display significant changes in FC galactosylation and fucosylation prior to the onset of RA. These changes towards a more pro-inflammatory phenotype could be involved in driving the disease process.

\section{INTRODUCTION}

Rheumatoid arthritis (RA) is an inflammatory disease characterised by chronic synovitis and erosive destruction of articular cartilage and bone. A subgroup of patients harbours antibodies targeting self-antigens. Among those, anticitrullinated protein antibodies (ACPA) exhibit remarkably high specificity for the disease and show a well-established association with a severe, erosive phenotype. Accumulating evidence suggests that ACPA could be involved in the disease process by enhancing inflammation and by specifically contributing to joint destruction through the activation of Fc-receptor positive cells including osteoclasts. ${ }^{1-5}$ Although the underlying mechanisms of this potential ACPA pathogenicity are incompletely understood, it is intriguing that ACPA can be present in asymptomatic individuals and in patients with joint pain (arthralgia) years before the onset of arthritis. ${ }^{6-9}$ In this early period, ACPA do not cause apparent pathology, indicating that, at a certain moment predisease, ACPA could acquire pathologic effector functions. ${ }^{10}$ This hypothesis of a quantitative and/or qualitative change of the citrulline-specific immune response is supported by an increase in ACPA levels, a spread in epitope recognition and isotype-usage as well as progressive avidity maturation before the onset of arthritis. ${ }^{11-14}$ Factors determining antibody effector functions could also be modified at this stage, eventually rendering ACPA pathogenic.

Early studies have shown that RA is associated with alterations in the glycosylation of serum IgG. ${ }^{15} 16$ Two N-linked, biantennary, complex-type carbohydrate structures are attached to the Fc part of $\mathrm{IgG}$, each located at a conserved position (Asn297) in the constant region of the heavy chains. These Fc-linked $\mathrm{N}$-glycans are often core-fucosylated and vary mainly in the number of galactose (Gal) residues, with the most prevalent glycoforms carrying no (G0), one (G1) or two galactose residues (G2). Additionally, a small proportion of the glycans may carry terminal sialic acid and/or bisecting $\mathrm{N}$-acetylglucosamine (GlcNAc) residues. The two Fc-linked carbohydrates are interposed between the heavy chains and are crucial for the three-dimensional structure and biological activity of the antibody. ${ }^{17}{ }^{18}$ Removal of the $\mathrm{Fc}$ glycans diminishes IgG Fc-mediated biological activity due to failure of the non-glycosylated molecule to bind to $\mathrm{F}_{\mathrm{c} \gamma}$-receptors (FcyR). ${ }^{19}{ }^{20}$ Likewise, lack of core fucose and presence of bisecting GlcNAc residues both enhance IgG-mediated antibodydependent cellular cytotoxicity (ADCC) due to a strong increase in affinity of the Fc-tail for Fc $\gamma$ RIIIIa. ${ }^{21-23}$ Additionally, Fc-linked glycans modulate activation of the complement cascade. ${ }^{24} 25$ Finally, more recent work demonstrated that terminal sialic acid and galactose residues can confer antiinflammatory properties to $\operatorname{IgG}$ by modulating its interaction with the lectins DC-SIGN and Dectin-1. ${ }^{26-28}$

In $\mathrm{RA}$, a lack of total $\mathrm{IgG} \mathrm{Fc}$ galactosylation correlates strongly with disease activity and severity, and reverses to normal levels upon effective treatment. ${ }^{29-34}$ Despite these associations, it has been 
difficult to determine how and to what extent IgG Fc glycosylation changes contribute to inflammation in RA, rather than being a non-specific biomarker. In a mouse model, adoptive transfer of enzymatically agalactosylated serum containing anti-collagen type II-IgG molecules from arthritic mice can cause arthritis of enhanced severity in the recipients. ${ }^{35}$ Also, removal of sialic acid from pooled human serum IgG abolishes its anti-inflammatory effects when infused into mice. ${ }^{28}$ These findings show that changes in IgG Fc glycosylation can indeed modulate inflammation in vivo, but data so far available are insufficient evidence to conclude on the pathogenic relevance of IgG Fc glycosylation in human RA.

More detailed information on this question could be obtained from studying events very early in the process of disease development. Currently, however, little information is available on changes of IgG Fc glycosylation in RA patients before disease onset, and no data have been put forward on autoantibodyspecific glycan profiles predisease. Based on these considerations, the pathogenic relevance of ACPA and our previous observation of ACPA-specific Fc glycosylation patterns in RA, ${ }^{36}$ we set out to study if (1) ACPA acquire specific Fc glycosylation patterns before the onset of arthritis, (2) if such qualitative changes predisease could predict disease development, and (3) if changes in ACPA Fc glycosylation occur prior to, after or simultaneously with systemic inflammation. Indeed, Fc glycosylation changes prior to detectable systemic inflammation and disease onset would support a model in which ACPA acquire pro-inflammatory properties driving the pathways that eventually result in arthritis.

\section{PATIENTS AND METHODS}

\section{Patients and controls}

Serum samples $(n=302)$ were obtained from ACPA-positive patients with arthralgia $(n=183)$ with a median ACPA serum titre of $419.0 \mathrm{U} / \mathrm{mL}$ (IQR 131.0-1216.0). For detailed patient characteristics see table 1 . Patients with arthralgia were followed over time, with serum samples and clinical parameters being collected at intervals up to the development of clinically overt arthritis (defined as the presence of one or more swollen joints), as previously described. ${ }^{8}$ From the arthralgia patients included at baseline, $n=105$ had developed arthritis within a median of 12 months (IQR 6-24) at the time the present study was undertaken. Patients with arthritis were classified as having either RA $(n=48)$ or undifferentiated arthritis (UA) $(n=57)$ based on the 1987 American College of Rheumatology (ACR) criteria. ${ }^{37}$ All other patients were considered to have persistent arthralgia. Control samples $(n=38)$ were obtained from healthy individuals age-matched and sex-matched to those arthralgia patients who developed arthritis with a time-interval of at least 13 months

\section{Table 1 Baseline characteristics of the study population}

\begin{tabular}{lll}
\hline Arthralgia patients & $\begin{array}{l}\text { Before QC } \\
\mathbf{n = 1 8 3}\end{array}$ & $\begin{array}{l}\text { After QC } \\
\mathbf{n}=172\end{array}$ \\
\hline Age in years, mean \pm SD & $48 \pm 11$ & $49 \pm 11$ \\
Female sex, $\mathrm{n}(\%)$ & $143(79)$ & $135(78)$ \\
Arthralgia duration in months, median (IQR) & $12(6-24)$ & $12(6-24)$ \\
Tender joint count (53 joints), median (IQR) & $0(0-3)$ & $0(0-3)$ \\
Rheumatoid factor positive, $\mathrm{n}(\%)$ & $86(48)$ & $79(46)$ \\
Follow-up, months (IQR) & $35(21-52)$ & $35(20-58)$ \\
Arthritis development, $\mathrm{n}(\%)$ & $105(58)$ & $99(57)$ \\
Development of RA, $\mathrm{n}(\%)$ & $48(26)$ & $43(25)$ \\
\hline QC, quality control. & &
\end{tabular}

between baseline and arthritis onset. All patients and controls gave informed consent for participation in the study.

\section{Isolation of IgG and ACPA-IgG from human serum}

Human polyclonal IgG molecules were isolated from $2 \mu \mathrm{L}$ of serum by affinity chromatography. ${ }^{38}$ ACPA were purified by antigen affinity chromatography from $25 \mu \mathrm{L}$ of serum using a modification of a pre-established protocol. ${ }^{36} 39$ Each serum sample was incubated for $1 \mathrm{~h}$ at $37^{\circ} \mathrm{C}$ on eight consecutive CCP2-coated ELISA wells (Immunoscan RA Mark 2, Eurodiagnostica) to obtain sufficient ACPA-IgG molecules. Following incubation with serum, each well was washed twice with phosphate buffered saline (PBS) and once with $25 \mathrm{mM}$ ammonium bicarbonate. ACPA were then eluted using $50 \mu \mathrm{L}$ of $100 \mathrm{mM}$ formic acid (pro analysi for mass spectrometry; Merck). All eight ACPA eluates were pooled, dried in a vacuum centrifuge and subjected to tryptic digest by adding $200 \mathrm{ng}$ trypsin (sequencing grade, Promega) in $40 \mu \mathrm{L}$ ammonium bicarbonate buffer, followed by overnight incubation at $37^{\circ} \mathrm{C}$.

\section{IgG glycopeptide profiling}

Trypsinised IgG $(250 \mathrm{~nL})$ and ACPA $(5 \mu \mathrm{L})$ molecules were separated and analysed on an Ultimate 3000 HPLC system (Dionex Corporation, Sunnyvale, California, USA) coupled to a quadrupole-TOF-mass spectrometry (MS) mass spectrometer (micrOTOF-Q, Bruker Daltonics, Bremen, Germany) as previously described. ${ }^{40}$ Briefly, tryptic glycopeptides were extracted by a C18 solid phase extraction trap column (Dionex Acclaim PepMap100) and separated on a Ascentis Express C18 nano liquid chromatography (LC) column (Supelco, Bellefonte, USA) as described. ${ }^{40}$ The HPLC was coupled to the MS by a sheath-flow electrospray ionization (ESI) interface. Quality of mass spectra was evaluated based on intensities of total IgG1 glycoforms. Following quality control based on signal-to-noise ratio of signal intensities of glycopeptide ions and verification of ACPA status, samples from 11 patients were excluded from the analysis. This did not change the overall composition of the study population (table 1). In total, $87.2 \%$ of samples remained for further analysis. The degree of galactosylation $(\mathrm{G})$, sialylation $(\mathrm{S})$, fucosylation $(\mathrm{F})$ and the frequency of bisecting $\mathrm{N}$-acetylglucosamine (GlcNAc, N) residues of IgG1 were calculated as described. ${ }^{40}$ Briefly, the following formulas were used: galactosylation $=(\mathrm{G} 1 \mathrm{~F}+\mathrm{G} 1 \mathrm{FN}+\mathrm{G} 1 \mathrm{FS}+\mathrm{G} 1 \mathrm{FNS}+\mathrm{G} 1$ $+\mathrm{G} 1 \mathrm{~N}+\mathrm{G} 1 \mathrm{~S}) * 0.5+\mathrm{G} 2 \mathrm{~F}+\mathrm{G} 2 \mathrm{FN}+\mathrm{G} 2 \mathrm{FS}+\mathrm{G} 2 \mathrm{FNS}+\mathrm{G} 2+\mathrm{G} 2 \mathrm{~N}$ $+\mathrm{G} 2 \mathrm{~S}$; fucosylation $=\mathrm{G} 0 \mathrm{~F}+\mathrm{G} 1 \mathrm{~F}+\mathrm{G} 2 \mathrm{~F}+\mathrm{G} 0 \mathrm{FN}+\mathrm{G} 1 \mathrm{FN}+\mathrm{G} 2 \mathrm{FN}$ plus; G1FS +G2FS; sialylation $=\mathrm{G} 1 \mathrm{FS}+\mathrm{G} 2 \mathrm{FS}+\mathrm{G} 1 \mathrm{FNS}+\mathrm{G} 2 \mathrm{FNS}$ $+\mathrm{G} 1 \mathrm{~S}+\mathrm{G} 2 \mathrm{~S}$; bisecting GlcNAc residues $=\mathrm{G} 0 \mathrm{FN}+\mathrm{G} 1 \mathrm{FN}$ $+\mathrm{G} 2 \mathrm{FN}+\mathrm{G} 1 \mathrm{FNS}+\mathrm{G} 2 \mathrm{FNS}+\mathrm{G} 0 \mathrm{~N}+\mathrm{G} 1 \mathrm{~N}+\mathrm{G} 2 \mathrm{~N}$.

\section{Statistical analysis}

Data analysis was performed using IBM SPSS Statistics V.20.0 and Graph Pad Prism V.5.01 software packages. Differences in relative abundances of glycoforms obtained were evaluated for statistical significance using the non-parametric Kruskal-Wallis test followed by Dunn's posthoc test to adjust for errors introduced by multiple testing. In case of comparison of only two groups, the Mann-Whitney test was used. This is indicated where appropriate.

\section{RESULTS}

\section{ACPA-specific Fc galactosylation profiles at baseline}

We first analysed Fc galactosylation of ACPA-IgG1 and total serum IgG1 in patients with arthralgia at baseline, that is, the moment of the patient's first presentation to the rheumatologist (figure 1A). To this end, we used a semi high-throughput procedure for antibody isolation followed by Fc N-glycopeptide 

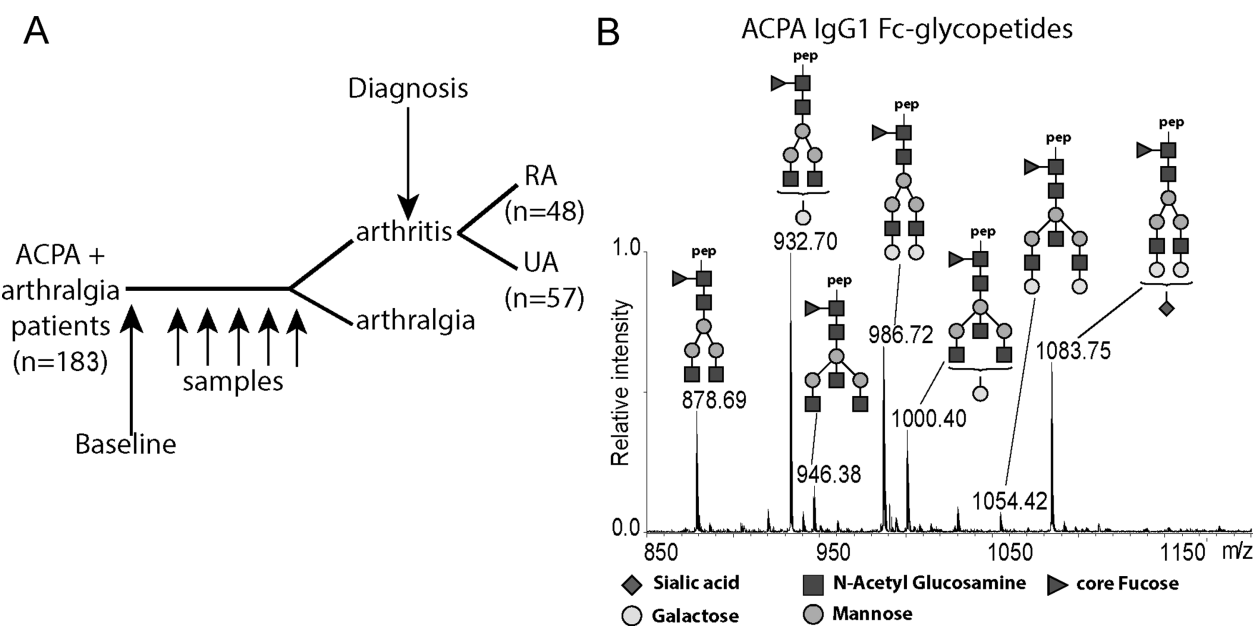

Figure 1 (A) Schematic depiction of the anticitrullinated protein antibodies (ACPA) positive arthralgia cohort used in the study; UA, undifferentiated arthritis, RA, rheumatoid arthritis. (B) Representative mass spectrum of ACPA IgG1 Fc glycopeptides isolated from an arthralgia patient; pep, peptide moiety; $\mathrm{m} / \mathrm{z}$, mass/charge.

profiling as outlined in figure 1B. Of note, this technique is not confounded by other glycans that might be linked to the IgG molecule, such as Fab-linked glycans. We report data on IgG1 Fc-glycopeptides in this study, as most patients harbour ACPA of the IgG1 subclass. ${ }^{41} 42$ Also, ACPA-IgG1 and total IgG1 showed much higher signal intensities of glycopeptide ions as compared to $\operatorname{IgG} 2$ and $\operatorname{IgG} 4$, suggesting predominant presence of the IgG1 isotype for ACPA and total IgG in our purified samples (data not shown).

At baseline, ACPA-IgG1 and total IgG1 exhibited a similar degree of $\mathrm{Fc}$ galactosylation (figure 2A). No difference in ACPA-IgG1 and total IgG1 Fc galactosylation was found between arthralgia patients who would later develop arthritis (figure 2B), nor was a baseline difference detected between patients developing UA or RA (figure 2C). Furthermore, no significant differences were found at baseline between these groups with regard to the frequency of sialylated glycoforms or glycoforms carrying bisecting GlcNAc residues (data not shown). The Fc-galactosylation profile of total IgG1 was comparable with that of age-matched and sex-matched healthy controls (data not shown). Taken together, ACPA-IgG1 and total IgG1 isolated from arthralgia patients showed a comparable Fc glycosylation profile at baseline, irrespective of future disease development.

\section{ACPA-specific Fc galactosylation profiles at the onset of arthritis}

Given the unaltered Fc galactosylation profiles at baseline, we next analysed whether the development of arthritis would be
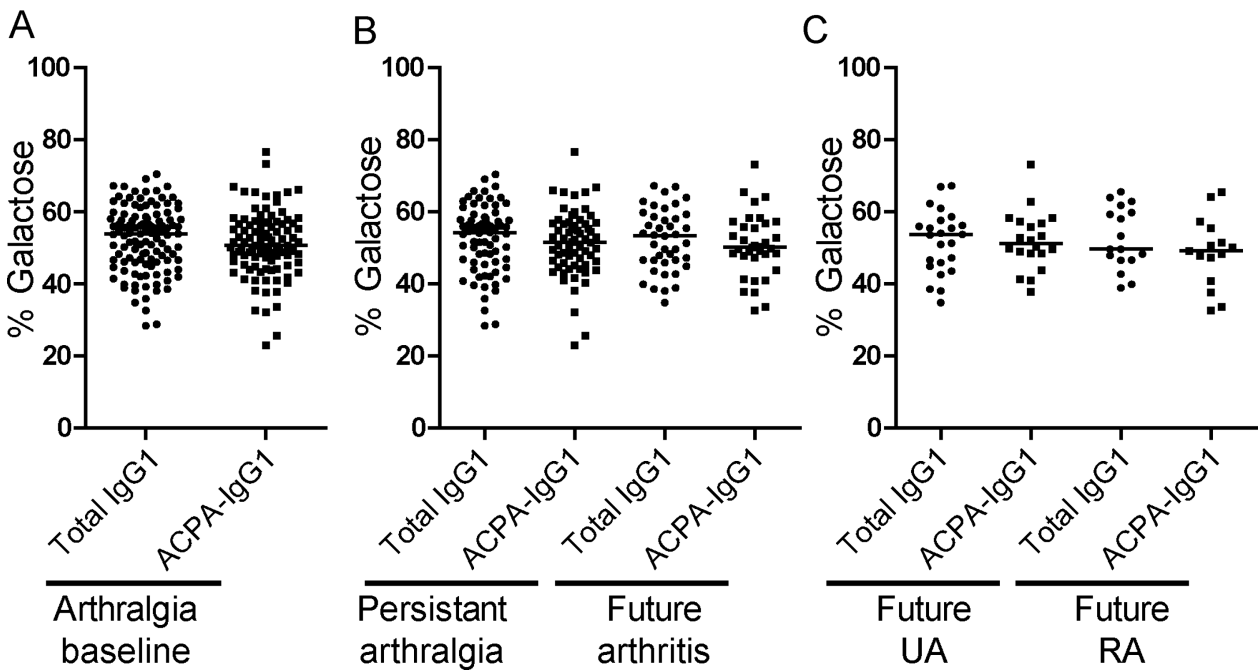

Figure 2 Fc galactosylation levels between ACPA-IgG1 and total IgG1 at baseline. (A) At baseline, similar Fc galactosylation levels were observed for total IgG1 and ACPA-IgG1 isolated from serum of arthralgia patients (median 53.9\% (IQR 46.1-58.7) for total IgG1 and 50.6\% (IQR 45.9-56.8) for ACPA-IgG1). Similarly, no significant differences were found for ACPA-lgG1 or total IgG1 Fc galactosylation between (B) patients presenting with persistent arthralgia and those who will develop arthritis (persistent arthralgia: median 54.3\% (IQR 46.1-58.5) for total IgG1 and 51.6\% (IQR 45.456.8) for ACPA-IgG1; future arthritis patients: $53.4 \%$ (IQR 46.3-59.0) for total IgG1 and 50.2\% (IQR 47.4-56.9) for ACPA-IgG1), or between (C) patients who will be affected by undifferentiated arthritis (UA) and those who will present with rheumatoid arthritis (RA) (future UA: median $53.7 \%$ (IQR 45.2-57.3) for total IgG1 and 51.3\% (IQR 48.5-57.3) for ACPA-IgG1; future RA: 49.7\% (IQR 60.9-46.6) for total IgG1 and 49.2\% (IQR 40.955.5) for ACPA-lgG1). Statistical analysis was performed using the non-parametric Mann-Whitney test for (A) and the non-parametric Kruskal-Wallis test followed by Dunn's posthoc test for (B and C). 
reflected by changes in Fc galactosylation on either ACPA-IgG1 or total serum IgG1. Of interest, patients presenting with symptoms classifying as RA, but not patients with UA, showed a significant reduction of ACPA-IgG1 Fc galactose residues when compared to baseline levels of arthralgia patients (figure 3A-C). This hypogalactosylation was also observed for total IgG1
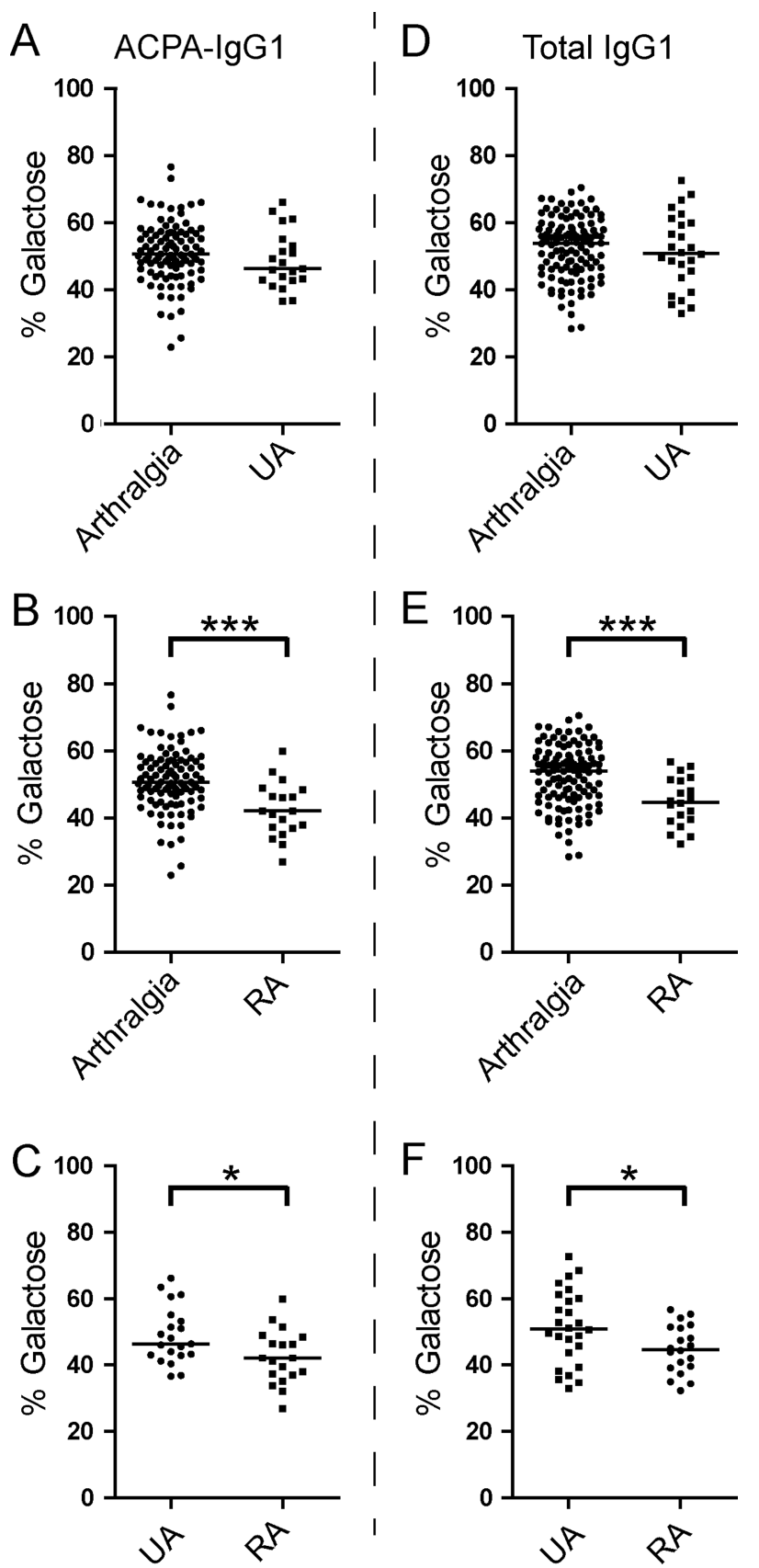

Figure 3 ACPA-lgG1 and total serum IgG1 Fc galactosylation profiles discriminate between rheumatoid arthritis (RA) and undifferentiated arthritis (UA) patients at the onset of arthritis. At the onset of arthritis, patients presenting with RA (B), but not those with UA (A), show a decrease of ACPA-IgG1 Fc galactosylation level compared to arthralgia patients at baseline (arthralgia at baseline: median 50.6\% (IQR 45.956.8); UA at diagnosis: $46.3 \%$ (IQR 42.9-54.1); RA at diagnosis: $42.1 \%$ (IQR 36.9-48.4)). (C) Accordingly, a significantly lower percentage of ACPA-lgG1 Fc galactosylation is observed in RA patients as compared to UA patients. $(D, E, F)$ A similar change was observed in the level of total IgG1 Fc galactosylation (arthralgia at baseline: median $53.9 \%$ (IQR 46.1-58.7); UA at diagnosis: 50.8\% (IQR 42.6-60.3); RA at diagnosis: 44.7\% (IQR 39.2-51.3)). Statistical differences were evaluated using the non-parametric Mann-Whitney test; * and *** represent a $p$ value $<0.05$ or a $p$ value $<0.0005$, respectively.

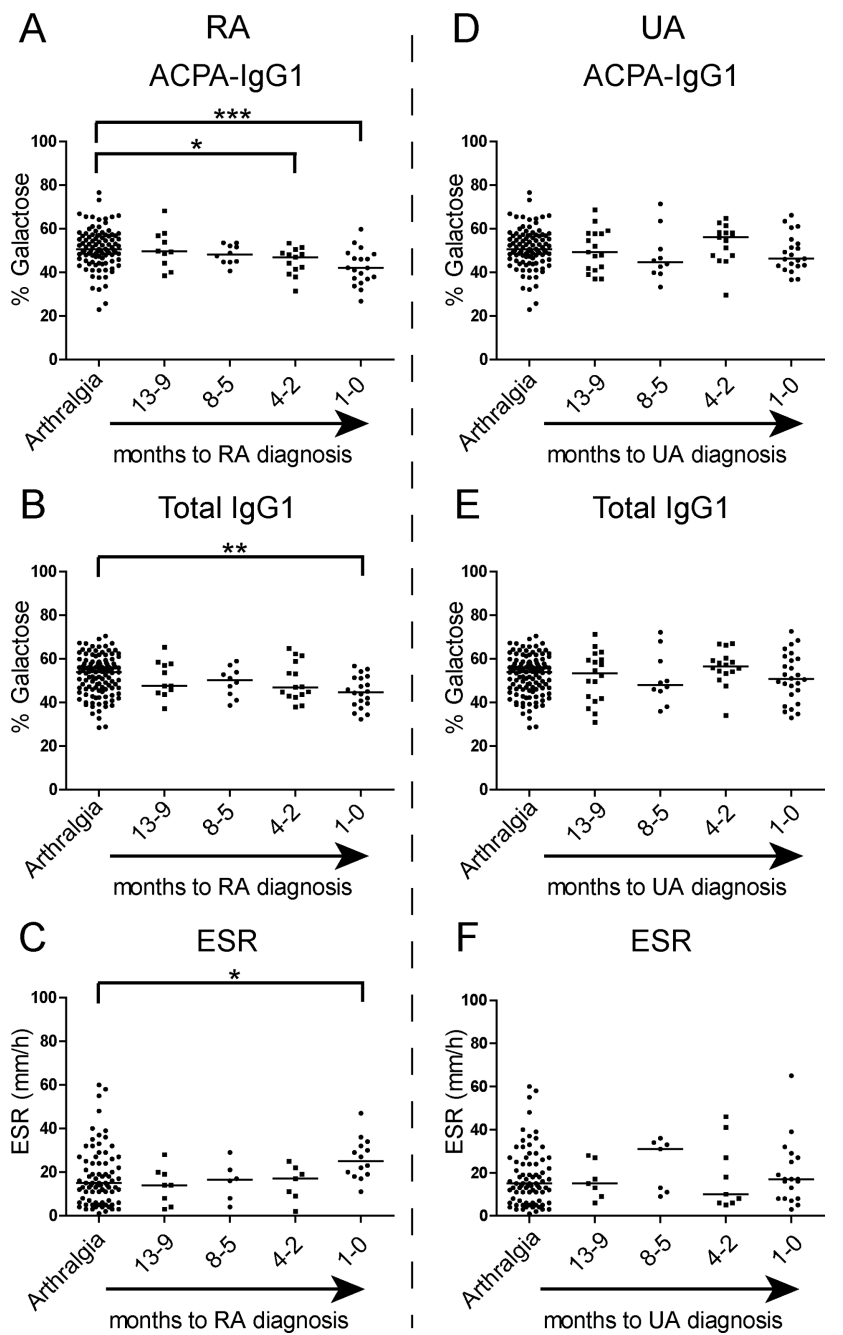

Figure 4 Changes in anticitrullinated protein antibodies (ACPA) Fc galactosylation precede the onset of rheumatoid arthritis (RA) and are paralleled by an increase in systemic inflammation. (A) ACPA-lgG1 Fc-specific galactosylation levels decrease predisease and become significantly different approximately 3 months $(-4$ to -2$)$ before the diagnosis of RA in comparison with arthralgia patients $>13$ months before diagnosis (arthralgia at baseline: median 50.6\% (IQR 45.9-56.8); -4 to -2 months pre-RA: $47.0 \%$ (IQR 40.4-49.7); RA at diagnosis: 42.1\% (IQR 36.9-48.4)). (B) For total IgG1, a significant decrease of Fc galactosylation content, albeit less pronounced, can only be observed at disease onset (arthralgia at baseline: median 53.9\% (IQR 46.1-58.7); RA at diagnosis: $44.7 \%$ (IQR 39.2-51.3)). (C) An increase in systemic inflammation, measured by erythrocyte sedimentation rate (ESR), is observed in parallel to the decrease of ACPA-IgG1 Fc galactosylation levels before the diagnosis of RA (arthralgia at baseline: median $15.0 \mathrm{~mm} / \mathrm{h}$ (IQR 7.0-25.0); RA at diagnosis: $25.0 \mathrm{~mm} / \mathrm{h}$ (IQR 18.832.5)). Patients who will develop undifferentiated arthritis (UA) show no significant variation over time in the percentage of (D) ACPA-IgG1 Fc galactosylation (arthralgia at baseline: 50.6\% (IQR 45.9-56.8); UA at diagnosis: 46.3\% (IQR 42.9-54.1)) and (E) total IgG1 Fc galactosylation levels (arthralgia at baseline: median 53.9\% (IQR 46.1-58.7); UA at diagnosis: $50.8 \%$ (IQR 42.6-60.3)) nor in (F) the ESR (arthralgia at baseline: $15.0 \mathrm{~mm} / \mathrm{h}$ (IQR 7.0-25.0); UA at diagnosis: $17.0 \mathrm{~mm} / \mathrm{h}$ (IQR 8.0-27.0)). The non-parametric Kruskal-Wallis test followed by Dunn's posthoc test was *, ** and ${ }^{* * *}$ represent $p$ values $<0.05,<0.005$ and $<0.0005$, respectively. 
(Figure 3D-F). Of note, we also observed a small but significant decrease of ACPA Fc-linked sialic acid content in RA patients as compared to arthralgia samples (see online supplementary figure S1B).

\section{Changes in ACPA Fc galactosylation precede disease onset in patients with $R A$}

Based on these findings, we aimed to determine whether the observed alterations in IgG1 Fc glycosylation precede the onset of arthritis. To this end, samples obtained up to 1 year $(-13$ to -9 months) prior to the onset of arthritis were analysed. One year prearthritis, Fc galactosylation of ACPA-IgG1 did not differ from baseline levels (figure 4A). However, patients who were classified as RA at the onset of arthritis showed a significant decrease of ACPA-IgG1 Fc galactosylation at around 3 months ( -4 to -2 months) prior to diagnosis (figure $4 \mathrm{~A}$ ). This predisease change of galactosylation was not noted for total IgG1 molecules, as a significant degree of hypogalactosylation could only be detected at the time of diagnosis (figure 4B). Patients presenting with UA, on the other hand, did not show significant alterations in ACPA-IgG1 and total IgG1 Fc galactosylation levels, neither predisease nor at the onset of arthritis (figure 4D,E). Importantly, no significant age difference was observed in the groups used for this analysis, excluding age as a confounder in the effects observed (data not shown). Taken together, these data indicate a reduction of galactose residues on ACPA-IgG1 shortly before disease onset in patients presenting with arthritis classifiable as RA.

\section{Fc galactosylation changes parallel increased systemic inflammation}

We previously reported a correlation between ACPA Fc galactosylation levels and systemic inflammation. ${ }^{36}$ As we now observed specific ACPA Fc galactosylation changes in the RA, but not in the UA group, we hypothesised that the RA group would show increased signs of inflammation. Indeed, an increase in erythrocyte sedimentation rate (ESR) was noted in the RA group, which paralleled the observed alterations in Fc galactosylation (figure 4C). Again, no such increase was observed in patients presenting with UA at the onset of arthritis (figure 4F), indicating a close correlation between changes in Fc galactosylation and the degree of systemic inflammation.

\section{ACPA-specific fucosylation patterns predisease}

Next to galactosylation and sialylation, the extent to which an antibody is core-fucosylated strongly influences its biological activity. While serum IgG1 generally displays a relatively high degree of fucosylation (>92\%), reduced fucosylation ( 42\%) has been found on antithrombocyte antibodies in immune thrombocytopenic purpura. ${ }^{43}$ Functionally, absence of core fucose residues leads to increased affinity of the $\mathrm{Fc}$ tail for binding to FcyRIIIa, thereby enhancing ADCC. ${ }^{21} 22$ A high degree of core fucosylation, on the other hand, decreases affinity for Fc $\gamma$ RIIIa and Fc $\gamma$ RIIB, thereby putatively allowing IgG molecules to bind to Fc $\gamma \mathrm{RI}^{22}{ }^{24}$ Given these functional consequences of core fucosylation, we extended our analysis to Fc-linked core fucose residues on ACPA-IgG1. Of interest, ACPA displayed a significantly higher degree of core fucosylation than total IgG1, both at baseline (arthralgia) and at the diagnosis of arthritis (Figure 5A-C). The degree of core fucosylation further increased in ACPA of RA patients towards the onset of arthritis (figure 6A), a phenomenon that we observed to a lesser degree also for total serum IgG1 (figure 6B). Again, no significant increase was observed for ACPA-IgG1 of patients presenting with UA (figure 6C,D).
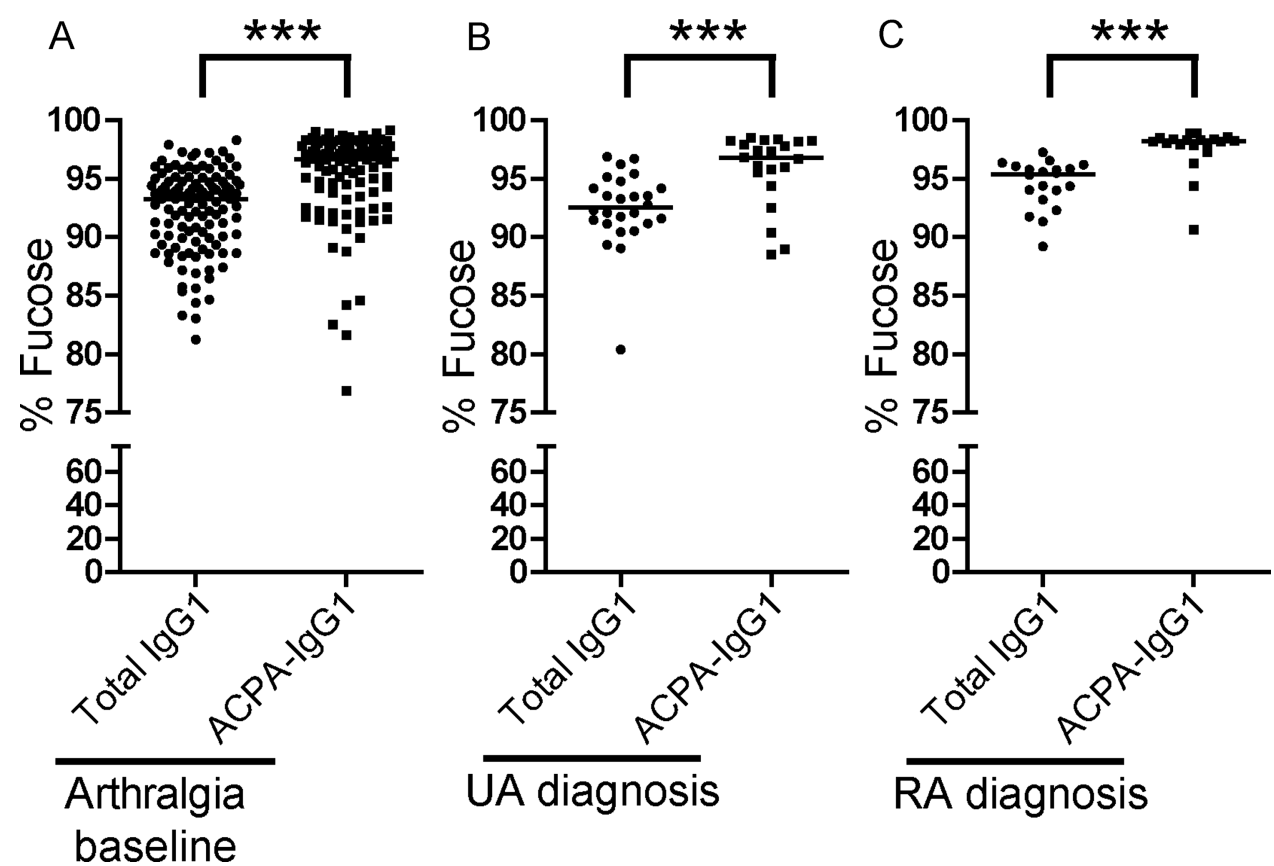

Figure 5 ACPA-specific fucosylation patterns at baseline and at the diagnosis of arthritis. ACPA-lgG1 present a significantly higher level of core fucosylation in (A) arthralgia patients at baseline and (B) in undifferentiated arthritis (UA) and (C) rheumatoid arthritis (RA) patients at diagnosis as compared to total serum IgG1 (arthralgia at baseline: median 93.3\% (IQR 89.9-94.8) for total IgG1 vs 96.6\% (IQR 93.8-97.8) for ACPA-IgG1; UA at diagnosis: 92.5\% (IQR 91.2-94.3) for total IgG1 vs 96.8\% (IQR 94.9-98.2) for ACPA-IgG1; RA at diagnosis: 95.4\% (IQR 93.4-96.0) for total IgG1 vs $98.2 \%$ (IQR 97.8-98.4) for ACPA-IgG1). Statistical differences were evaluated using the non-parametric Mann-Whitney test. *** represents $p$ value $<0.0005$ 
Figure 6 Changes of ACPA-IgG1 and total IgG1 core fucosylation precede the diagnosis of rheumatoid arthritis (RA). (A) Although already high at baseline, the percentage of core fucosylation slightly increases in ACPA-IgG1 before RA onset in comparison with that of arthralgia patients at baseline (arthralgia at baseline: median 96.6\% (IQR 93.897.8); RA at diagnosis: $98.2 \%$ (IQR 97.8-98.4)). (B) These changes were also observed for total $\lg \mathrm{G} 1$, albeit less pronounced (arthralgia at baseline: median 93.3\% (IQR 89.9-94.8); RA at diagnosis: 95.4\% (IQR 93.4-96.0)). No significant variation in (C) ACPA-IgG1 and (D) total IgG1 core fucosylation levels was observed in patients who develop undifferentiated arthritis (UA) as compared to arthralgia patients at baseline (arthralgia at baseline: median 96.6\% (IQR 93.8-97.8) for ACPA-lgG1 and 93.3\% (IQR 89.994.8) for total IgG1; UA at diagnosis: 96.8\% (IQR 94.9-98.2) for ACPA-IgG1 and 92.5\% (IQR 91.2-94.3) for total lgG1). The non-parametric KruskalWallis test followed by Dunn's posthoc test was used, * and ** represent $p$ values $<0.05$ and $p$ values $<0.005$, respectively.
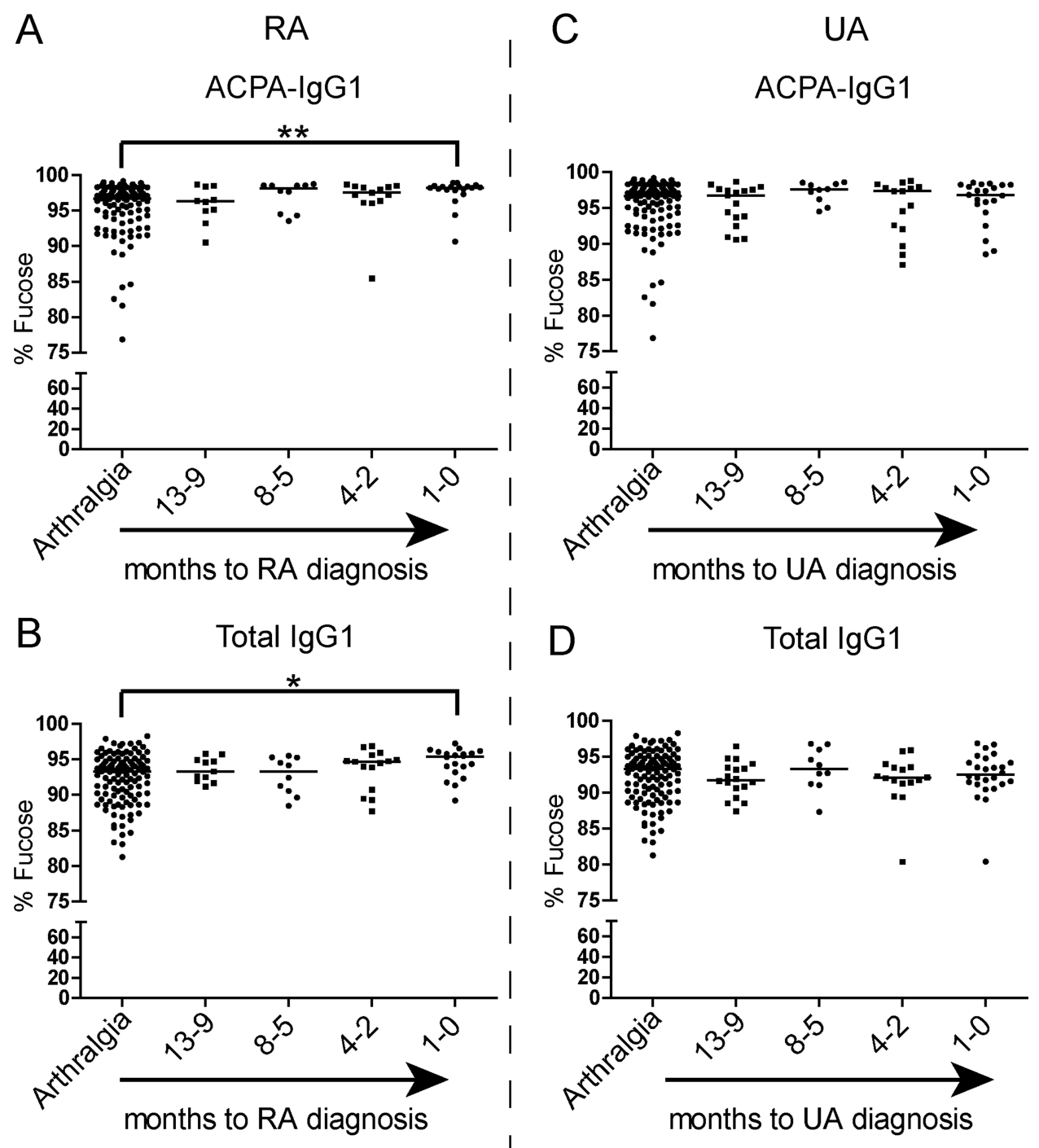

\section{DISCUSSION}

Next to well-established clinical associations, accumulating experimental evidence points to a pathogenic role of ACPA in RA pathogenesis. ${ }^{1-5}$ However, ACPA can be present in serum for many years in subjects that do not show clinical or serologic signs of inflammation. ${ }^{6} 7810$ These observations indicate that a yet unknown trigger could induce a quantitative and/or qualitative change in the ACPA response prior to disease onset. Here, we hypothesised that such a change could be related to ACPA-IgG Fc glycosylation based on the observation that the monosaccharide composition of Fc glycans can strongly influence the inflammatory activity of IgG. ${ }^{45} 46$ Indeed, employing a robust technique of IgG Fc-specific glycopeptide profiling, we found a decrease of galactosylation and an increase of core fucosylation of serum ACPA-IgG1 shortly before the onset of RA. Based on published data, these features are thought to enhance the overall proinflammatory effector functions of IgG molecules. ${ }^{22} 4546$

So far, reports on Fc glycosylation changes of total serum IgG in individuals prior to the development of RA have been limited and conflicting. Comparison of $\operatorname{IgG}$ glycosylation in patients with early onset synovitis who developed RA, or other inflammatory joint diseases within 2 years, revealed an elevated level of agalactosylated IgG (G0 glycoforms) in patients with future RA. ${ }^{47}$ Another study suggested a significant increase of IgG G0 glycoforms in patients with RA around 3.5 years before the onset of disease. ${ }^{48}$ By contrast, others reported no significant differences in $\operatorname{IgG}$ galactosylation levels between recent onset RA patients and healthy donors. ${ }^{31}$ Of note, these studies analysed total IgG and not specific autoantibodies and applied different analytical techniques which, in part, do not differentiate between IgG subclasses or Fc-linked and Fab-linked glycans. Additional differences might originate from the cohorts studied and the definitions used for patient classification. Our present study, albeit limited by comparatively low patient numbers, indicates that ACPA-IgG1 Fc glycosylation changes occur only within months before the onset of arthritis. Nonetheless, these changes were detected several months before comparable changes could be observed for total IgG1.

The Fc glycosylation changes described were only observed in patients presenting with disease symptoms classifiable as RA according to the 1987 ACR criteria, but not in patients with 1987-UA. ${ }^{37}$ As stated in the discussion of the ACR/EULAR 2010-criteria' manuscript, use of the 1987 criteria is advised for pathogenetic studies. ${ }^{49}$ By focussing on these criteria, we had the possibility to choose defined subgroups of patients that differ in the degree of their inflammatory phenotype. UA patients represent the less inflammatory subgroup (lower number of swollen joints, less systemic inflammation (ESR)), even though, mainly due to the presence of ACPA, the vast majority of these patients would classify as RA following the 2010 ACR/EULAR criteria. ${ }^{49}$ Separating 
the groups in 1987-UA and 1987-RA allowed us to show that Fc glycosylation changes on ACPA-IgG1 indeed occur prior to RA onset. Although not useful for diagnostic purposes, this observation could be informative with respect to pathogenetically relevant inflammatory processes pre-RA.

In this context, our data underline the close correlation between systemic inflammation and ACPA-IgG1 Fc glycosylation. In fact, an increase in systemic inflammation directly paralleled the decrease of ACPA Fc-linked galactose residues (figure 4C). Based on this observation, it is not possible to determine whether ACPA glycosylation changes are cause or consequence of inflammation. However, it is intriguing to note that the decrease in ACPA Fc galactosylation preceded that of total serum IgG. This could be due to differences in compartments in which the respective antibodies are produced. It is possible that agalactosylated ACPA in serum originate mainly from synovial tissue or other sites of preclinical inflammation. ${ }^{50}$ Supporting this view, we have previously found a high degree of IgG1-G0 glycoforms specifically on ACPA in synovial fluid, but not on total IgG1. ${ }^{36}$ Together with the potential presence of subclinical inflammation in synovium prior to the onset of clinically overt arthritis, leakage of synovial ACPA to the serum compartment could explain the findings observed. Additionally, it would emphasise the role of the B-cell microenvironment for Fc glycosylation, in line with our previous finding that various extracellular factors can modulate Fc glycosylation patterns in vitro. ${ }^{51}$

Finally, next to changes in Fc-linked galactosylation, we observed that ACPA-IgG1 display a higher frequency of core fucose residues compared with total serum IgG1. This feature was already detectable at baseline, underlining the notion of $\mathrm{B}$ cell-specific regulation of Fc-linked glycan profiles. The high degree of core fucosylation further increased towards the onset of arthritis at a time-course comparable to the loss of galactose residues. Again, this observation was only found in patients with RA. The biological relevance of this finding is yet unclear but indicates a reduced affinity of ACPA for FcyRIIIa and Fc $\gamma$ RIIb, but not for other activating FcyR, such as the high-affinity

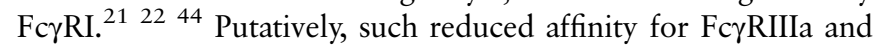
FcrRIIb could increase availability of ACPA for binding to Fc $\gamma$ RI, thereby favouring immune activating effects.

Taken together, the present study shows that ACPA-IgG1 molecules acquire changes in Fc glycosylation shortly before the onset of arthritis. These changes could be involved in driving the inflammatory disease process, although the underlying mechanisms remain yet to be defined.

Acknowledgements We thank Carolien Koeleman for excellent technical support.

Contributors YR acquired data, analysed, interpreted data, drafted and revised the paper. EE and MHS acquired data, analysed and revised the draft paper. LAT, LvdS and DS collected cohort samples and revised the draft paper. AMD and TWJH revised the draft paper. MW designed experiments and revised the draft paper. REMT and HUS designed experiments, interpreted data, drafted and revised the paper.

Funding This work was supported by grants from the Dutch Arthritis Foundation (NR 10-1-204), the European Union (Seventh Framework Programme integrated project Masterswitch, grant number: 223404, and HighGlycan, grant number: 278535) and the IMI EU funded project BeTheCure (contract no 115142-2). REMT is recipient of a NWO-ZonMW VICI grant. MHJS is supported by Hoffmann La Roche. LAvdS and DvS were supported by the Dutch Arthritis Association (No. 8-1-306). LT is supported by a NWO-ZonMW Vidi grant and by a fellowship from Janssen Biologics.

Competing interests None.

Ethics approval This study was approved by the local ethics committee, and all participants gave informed consent.

Provenance and peer review Not commissioned; externally peer reviewed.

\section{REFERENCES}

1 Kuhn KA, Kulik L, Tomooka B, et al. Antibodies against citrullinated proteins enhance tissue injury in experimental autoimmune arthritis. J Clin Invest 2006;116:961-73.

2 Clavel C, Nogueira L, Laurent L, et al. Induction of macrophage secretion of tumor necrosis factor alpha through Fcgamma receptor lla engagement by rheumatoid arthritis-specific autoantibodies to citrullinated proteins complexed with fibrinogen. Arthritis Rheum 2008:58:678-88.

3 Sokolove J, Zhao X, Chandra PE, et al. Immune complexes containing citrullinated fibrinogen costimulate macrophages via Toll-like receptor 4 and Fcgamma receptor. Arthritis Rheum 2011;63:53-62.

4 Trouw LA, Haisma EM, Levarht EW, et al. Anti-cyclic citrullinated peptide antibodies from rheumatoid arthritis patients activate complement via both the classical and alternative pathways. Arthritis Rheum 2009;60:1923-31.

5 Harre U, Georgess D, Bang H, et al. Induction of osteoclastogenesis and bone loss by human autoantibodies against citrullinated vimentin. J Clin Invest 2012;122:1791-802.

6 Nielen MM, van Schaardenburg D, Reesink HW, et al. Specific autoantibodies precede the symptoms of rheumatoid arthritis: a study of serial measurements in blood donors. Arthritis Rheum 2004;50:380-6.

7 Rantapaa-Dahlqvist S, de Jong BA, Berglin E, et al. Antibodies against cyclic citrullinated peptide and IgA rheumatoid factor predict the development of rheumatoid arthritis. Arthritis Rheum 2003;48:2741-9.

8 Bos WH, Wolbink GJ, Boers M, et al. Arthritis development in patients with arthralgia is strongly associated with anti-citrullinated protein antibody status: a prospective cohort study. Ann Rheum Dis 2010;69:490-4.

9 Limper M, van de Stadt L, Bos W, et al. The acute-phase response is not predictive for the development of arthritis in seropositive arthralgia - a prospective cohort study. J Rheumatol 2012;39:1914-17.

10 van de Sande MG, de Hair MJ, van der Leij C, et al. Different stages of rheumatoid arthritis: features of the synovium in the preclinical phase. Ann Rheum Dis 2011;70:772-7.

11 Suwannalai $P$, van de Stadt LA, Radner $H$, et al. Avidity maturation of ACPA in rheumatoid arthritis. Arthritis Rheum 2012;64:1323-8.

12 van de Stadt LA, de Koning MH, van de Stadt RJ, et al. Development of the anti-citrullinated protein antibody repertoire prior to the onset of rheumatoid arthritis. Arthritis Rheum 2011;63:3226-33.

13 van der Woude D, Rantapaa-Dahlqvist S, Ioan-Facsinay A, et al. Epitope spreading of the anti-citrullinated protein antibody response occurs before disease onset and is associated with the disease course of early arthritis. Ann Rheum Dis 2010;69:1554-61.

14 Ioan-Facsinay A, Willemze A, Robinson DB, et al. Marked differences in fine specificity and isotype usage of the anti-citrullinated protein antibody in health and disease. Arthritis Rheum 2008;58:3000-8.

15 Parekh RB, Dwek RA, Sutton BJ, et al. Association of rheumatoid arthritis and primary osteoarthritis with changes in the glycosylation pattern of total serum IgG. Nature 1985:316:452-7.

16 Parekh $R$, Isenberg $D$, Rook $G$, et al. A comparative analysis of diseaseassociated changes in the galactosylation of serum IgG. J Autoimmun 1989;2:101-14

17 Krapp S, Mimura Y, Jefferis R, et al. Structural analysis of human IgG-Fc glycoforms reveals a correlation between glycosylation and structural integrity. J Mol Biol 2003;325:979-89.

18 Yamaguchi $Y$, Nishimura $M$, Nagano $M$, et al. Glycoform-dependent conformational alteration of the Fc region of human immunoglobulin $\mathrm{G} 1$ as revealed by NMR spectroscopy. Biochim Biophys Acta 2006;1760:693-700.

19 Jung ST, Reddy ST, Kang TH, et al. Aglycosylated IgG variants expressed in bacteria that selectively bind Fcgamma RI potentiate tumor cell killing by monocyte-dendritic cells. Proc Natl Acad Sci USA 2010;107:604-9.

20 Mimura Y, Church S, Ghirlando R, et al. The influence of glycosylation on the thermal stability and effector function expression of human IgG1-Fc: properties of a series of truncated glycoforms. Mol Immunol 2000;37:697-706.

21 Ferrara C, Grau S, Jager C, et al. Unique carbohydrate-carbohydrate interactions are required for high affinity binding between FcgammaRIII and antibodies lacking core fucose. Proc Natl Acad Sci USA 2011;108:12669-74.

22 Shields RL, Lai J, Keck R, et al. Lack of fucose on human IgG1 N-linked oligosaccharide improves binding to human Fcgamma RIII and antibody-dependent cellular toxicity. J Biol Chem 2002;277:26733-40.

23 Shinkawa T, Nakamura K, Yamane N, et al. The absence of fucose but not the presence of galactose or bisecting $\mathrm{N}$-acetylglucosamine of human IgG1 complex-type oligosaccharides shows the critical role of enhancing antibody-dependent cellular cytotoxicity. J Biol Chem 2003;278:3466-73.

24 Malhotra R, Wormald MR, Rudd PM, et al. Glycosylation changes of IgG associated with rheumatoid arthritis can activate complement via the mannose-binding protein. Nat Med 1995; 1:237-43.

25 Raju TS. Terminal sugars of Fc glycans influence antibody effector functions of IgGs. Curr Opin Immunol 2008;20:471-8. 
26 Anthony RM, Kobayashi T, Wermeling F, et al. Intravenous gammaglobulin suppresses inflammation through a novel T(H)2 pathway. Nature 2011;475:110-13.

27 Karsten CM, Pandey MK, Figge J, et al. Anti-inflammatory activity of IgG1 mediated by Fc galactosylation and association of Fcgamma RIIB and dectin-1. Nat Med 2012;18:1401-6.

28 Kaneko Y, Nimmerjahn F, Ravetch JV. Anti-inflammatory activity of immunoglobulin $G$ resulting from Fc sialylation. Science 2006;313:670-3.

29 van Zeben D, Rook GA, Hazes JM, et al. Early agalactosylation of lgG is associated with a more progressive disease course in patients with rheumatoid arthritis: results of a follow-up study. Br J Rheumatol 1994;33:36-43.

30 Lacki JK, Porawska W, Mackiewicz U, et al. Changes in agalactosyl IgG levels correlate with radiological progression in early rheumatoid arthritis. Ann Med 1996:28:265-9.

31 Gindzienska-Sieskiewicz E, Klimiuk PA, Kisiel DG, et al. The changes in monosaccharide composition of immunoglobulin $\mathrm{G}$ in the course of rheumatoid arthritis. Clin Rheumatol 2007;26:685-90.

32 Croce A, Firuzi O, Altieri F, et al. Effect of infliximab on the glycosylation of lgG of patients with rheumatoid arthritis. J Clin Lab Anal 2007;21:303-14.

33 Pasek M, Duk M, Podbielska $M$, et al. Galactosylation of IgG from rheumatoid arthritis (RA) patients - changes during therapy. Glycoconj J 2006:23:463-71.

34 Van Beneden K, Coppieters K, Laroy W, et al. Reversible changes in serum immunoglobulin galactosylation during the immune response and treatment of inflammatory autoimmune arthritis. Ann Rheum Dis 2009;68:1360-5.

35 Rademacher TW, Williams P, Dwek RA. Agalactosyl glycoforms of IgG autoantibodies are pathogenic. Proc Natl Acad Sci USA 1994;91:6123-7.

36 Scherer HU, van der Woude D, loan-Facsinay A, et al. Glycan profiling of anti-citrullinated protein antibodies isolated from human serum and synovial fluid. Arthritis Rheum 2010;62:1620-9.

37 Arnett FC, Edworthy SM, Bloch DA, et al. The American Rheumatism Association 1987 revised criteria for the classification of rheumatoid arthritis. Arthritis Rheum 1988;31:315-24

38 Selman MH, McDonnell LA, Palmblad M, et al. Immunoglobulin G glycopeptide profiling by matrix-assisted laser desorption ionization Fourier transform ion cyclotron resonance mass spectrometry. Anal Chem 2010;82:1073-81.
39 Scherer HU, Wang J, Toes RE, et al. Immunoglobulin 1 (IgG1) Fc-glycosylation profiling of anti-citrullinated peptide antibodies from human serum. Proteomics Clin Appl 2009;3:106-15.

40 Selman MH, Derks RJ, Bondt A, et al. Fc specific IgG glycosylation profiling by robust nano-reverse phase HPLC-MS using a sheath-flow ESI sprayer interface. J Proteomics 2012;75:1318-29.

41 Engelmann R, Brandt J, Eggert $M$, et al. $\lg G 1$ and $\lg G 4$ are the predominant subclasses among auto-antibodies against two citrullinated antigens in RA. Rheumatology (Oxford) 2008;47:1489-92.

42 Chapuy-Regaud S, Nogueira L, Clavel C, et al. IgG subclass distribution of the rheumatoid arthritis-specific autoantibodies to citrullinated fibrin. Clin Exp Immunol 2005; 139:542-50.

43 Wuhrer M, Porcelijn L, Kapur R, et al. Regulated glycosylation patterns of IgG during alloimmune responses against human platelet antigens. J Proteome Res 2009;8:450-6.

44 Siberil S, de Romeuf C, Bihoreau N, et al. Selection of a human anti-RhD monoclonal antibody for therapeutic use: impact of IgG glycosylation on activating and inhibitory Fc gamma R functions. Clin Immunol 2006;118:170-9.

45 Arnold JN, Wormald MR, Sim RB, et al. The impact of glycosylation on the biological function and structure of human immunoglobulins. Annu Rev Immunol 2007;25:21-50

46 Zauner G, Selman MH, Bondt A, et al. Glycoproteomic analysis of antibodies. Mol Cell Proteomics 2013:12:856-65.

47 Young A, Sumar N, Bodman K, et al. Agalactosyl IgG: an aid to differential diagnosis in early synovitis. Arthritis Rheum 1991;34:1425-9.

48 Ercan A, Cui J, Chatterton DE, et al. Aberrant IgG galactosylation precedes disease onset, correlates with disease activity, and is prevalent in autoantibodies in rheumatoid arthritis. Arthritis Rheum 2010;62:2239-48.

49 Aletaha D, Neogi T, Silman AJ, et al. 2010 Rheumatoid arthritis classification criteria: an American College of Rheumatology/European League Against Rheumatism collaborative initiative. Ann Rheum Dis 2010;69:1580-8.

50 Makrygiannakis D, Hermansson M, Ulfgren AK, et al. Smoking increases peptidylarginine deiminase 2 enzyme expression in human lungs and increases citrullination in BAL cells. Ann Rheum Dis 2008;67:1488-92.

51 Wang J, Balog $\mathrm{Cl}$, Stavenhagen $\mathrm{K}$, et al. Fc-glycosylation of $\lg \mathrm{G} 1$ is modulated by B-cell stimuli. Mol Cell Proteomics 2011;10:M110.004655. 